Current Research in Psychology 1 (1): 35-52, 2010

ISSN 1949-0178

(C) 2010 Science Publications

\title{
Object Identity, Apparent Motion, Transformation Geometry
}

\author{
Felice L. Bedford and Birgitta E. Mansson \\ Department of Psychology, University of Arizona, Tucson AZ 85721
}

\begin{abstract}
Problem statement: A classic question in cognition was addressed on how two glimpses separated in time are determined to refer either to the same object or to two different objects, without which coherent perception of the world is not possible. Approach: A general solution was offered in which observers exploit an entire family of geometries. It was argued that a geometry hierarchy is an attractive candidate for a solution because it is can be used on glimpses not only from different times, but from different spatial locations, different eyes, and different modalities. The theory was applied to the phenomenon of apparent motion, a time-honored paradigm for uncovering rules of object or numerical identity. Results: It was found that there was a preference to map a figure onto the "same form" (isometric transformation) when available (Exp. 1), despite controversy about form and apparent motion in the literature. When the same form was not available, next preferred was a similarity transformation ("different size"; Exps. 2 and 3) when the perceptual system was given a choice. Finally, it was found that a figure even matched to an extreme topological transformation ("plastic deformation"; Exp. 4) when that was the lowest-level geometric choice available. Conclusions/Recommendations: The experiments supported a hierarchical structure of unconscious perceptual preferences in object identity which importantly explains how the exact same pair of stimuli can sometimes be judged to refer to the same object, but sometimes not. It was also suggested that the inherent flexibility of this solution resolves contradictions in the literature concerning the relevance of form in apparent motion and that the hierarchy satisfyingly captures intuitions about what is "similar" in perception while pointing to dangers of relying exclusively on such intuition.
\end{abstract}

Key words: Cognition, perception, mathematics, numerical identity, similarity, topology

\section{INTRODUCTION}

Object identity, apparent motion, transformation geometry: A squirrel runs behind a tree and moments later a squirrel emerges. Is it the exact same squirrel or merely a cousin? Achieving numerical or object identity-knowing whether or not the two glimpses do or do not refer to the same object-is a challenge for observers at all processing levels. Frequent movement of observers virtually guarantees that a single object will lead to different retinal projections from one instant in time to the next. For instance, the same parked car viewed head on casts a different image than the car viewed from an angle. Likewise, changes to the objects themselves produce glimpses that are not identical. The car can be driven from one location to the next; the squirrel can be crouching before darting behind the tree, yet emerge on its hind legs. Human observers are remarkably good at knowing when we have encountered the exact same object even though we rarely have the benefit of the exact same stimulus, either rationally, perceptually or cognitively. The issue of object identity is a classic one in perception and philosophy.
One approach for uncovering the fundamental rules of object identity is based on development. If relevant knowledge is available early, then in some sense it is a primary solution to identity. Elizabeth Spelke and colleagues (Spelke et al., 1995; Spelke and van de Walle, 1993) have argued that even very young infants know that objects travel on paths that are continuous in space and in time such that violations are informative of numerosity. For instance, if a green ball goes behind a screen and a green ball comes out another screen that is separated from the first, then 3 month old infants will behave as if there are two green balls. They have suggested this is because of early knowledge that one object cannot not magically jump the gap between the screens. Older infants know that a member of one category of object (e.g., elephant) cannot turn into another (e.g., truck) even there is only single screen without a spatial gap, behind which one enters and the other emerges (Xu and Carey, 1996; Wiggins, 1980). Knowledge about properties specific to some categories of objects but not others allow more refined judgments (Narter, 1998). If you return home after a month, a bigger squirrel in your yard could be the same squirrel,

Corresponding Author: Felice L. Bedford, Department of Psychology, University of Arizona, Tucson AZ 85721 
but a bigger car in your garage could not be. Even more detailed knowledge can be useful in special situations. Teresa Wilcox (1999) has argued that instructions about an "electric warmer" hidden behind a screen that turns green objects into red ones changes will change identity outcomes for young observers. Children will now believe that a green ball that disappeared behind that screen is the same ball as a red one that emerged from the other side, but would not without those instructions.

Developmentally relevant solutions to object identity emerged in consideration of a single problem: identity across gaps in time, often spatio-temporal gaps. However, consideration that the problem of object identity is much broader than this prototypic situation points to a different kind of fundamental solution for object identity. Bedford $(2001 ; 1999 ; 2007)$ argues that the same object identity problem must be solved not only for samples that come from different times, but also when the samples do not come from different times at all. That is, samples can also come from different modalities, eyes, or spatial locations. How do you know that the pen you are seeing and the pen you feeling are the exact same pen? Or that the pen you are feeling and the cup of coffee you are eyeing are different objects? Asked generally, the question is how does the observer determine when any two non-identical samples, s1 and 2 , arise from the same object? Bedford argues object identity may play a role in nearly all perceptual accomplishments, including tracking objects over time, cross-modal perception, stereopsis and perceptual organization across space, as well as in laboratory phenomena of apparent motion, prism adaptation, ventriloquism, priming and Gestalt grouping, since much of perceiving the world involves taking in information from non-identical samples.

The same formal problem in different domains invites the possibility of a common solution (Dennett, 1996). Task-specific knowledge of electric warmers, or even that animals and plants can grow, can be used for identity but they are not applicable to all domains and thus cannot constitute the core solution to object identity. Categorization of objects into their kinds (elephants/trucks) also cannot be relied upon exclusively because object identity is achieved even when we cannot recognize the objects involved (Bloom, 2000). Spatial-temporal continuity is general, but often that is the problem, not the solution: Did discrete samples s1 and 2 result from a singe object continuously transforming from one state to the other (What may appear to be clear violations of spatialtemporal continuity are problematic in another way. They do not necessarily imply that observers will deduce two objects rather than one. For instance, in apparent motion, as will be)?
The core of object identity will consist of criteria that are applicable to all domains with the same formal structure. The purpose of the present article is to test the applicability of a potentially general set of core criteria for object identity to the phenomenon of apparent motion. First the role of identity in apparent motion will be noted, then the proposed criteria will be introduced, followed by the predictions of the criteria in the apparent motion paradigm and finally by the experiments.

Apparent motion: Apparent motion is a classic (Wertheimer, 1961; Korte, 1915) readily manipulable laboratory phenomenon that is well-accepted to depend upon an object identity judgment (e.g., Rock, 1983; Shepard, 1984; Kahneman et al., 1992; Ullman, 1979; Warren, 1977; Xu and Carey, 1996). As such, it is an ideal candidate for testing the criteria of object identity. In apparent motion, a stimulus is shown in one location for a brief period of time and is replaced by a second stimulus in a different location a short time later. The sequence is usually repeated a number of times and observers see a stimulus moving back and forth between the two locations. Rock (1983) and Shepard (1984) argue that although intermediate positions are not detected on the retina, an interpretation of a single object moving back and forth between the two locations is preferable to accepting the coincidence that two identical or similar looking objects are appearing and disappearing in alternation. The phenomenon hinges on misidentifying the two stimuli as two different glimpses of the same object at two different times. If the two samples are judged to refer to one object, one object in motion is experienced. If the two samples are judged to refer to two objects, then two objects flashing on and off are correctly seen and there is no impression of motion.

A compelling demonstration that destroying the one-object assumption destroys apparent motion comes from Sigman and Rock (1974); Rock, (1983). At one time, there is a small black dot in position A and a big screen covering position $\mathrm{B}$ and a short time later, the screen covers position A and there is a small black dot in position B. Observers do not see the small black dot moving from A to B, as they would without the screen. Rather, the display looks as if there is a screen moving back and forth to successively occlude and reveal two dots, one on each side. The addition of the screen invites a different explanation of the mysterious sensory appearance and disappearance of two dot stimuli: Rather than one object moving back and forth, a screen is moving back and forth in front of two objects. Consequently, apparent motion can be used as tool to uncover criteria of identity. In its simplest form, if two stimuli do not satisfy the criteria that they result from the same object, then apparent motion between those two stimuli will not occur (Chen, 1985; Warren, 1977). 
Transformation geometry: The potentially general set of criteria comes from geometry. All objects have extended contours-form-and geometry is the formal study of form. Point sources can be considered degraded forms. In addition to space, geometry can be applied to time by considering temporally extended contours analogous to spatially extended contours (Bedford, 2001). The generality is furthered assured by using a whole family of increasingly broad geometries that fit within one another like set of Russian nesting dolls. Euclidean geometry is the smallest, most familiar of these geometries, which contains properties such as size, angle and parallelism and usually captures our intuitions about the way the world works. Yet there is reason to believe that other geometries may be part of our mental constitution, even if they are largely inaccessible to conscious introspection.

To create broader geometries mathematically, there are two approaches, axiomatic and transformational. It is interesting to note that these two complementary approaches in mathematics, which lead to the same conclusion by different routes, mirror what have been competing approaches in the history of perception. What is effectively an axiomatic approach in perception calls attention to static properties of an image (Helmholtz, 2005), whereas the transformation approach emphasizes properties that remain invariant in the face of change (Gibson, 1966). In the axiomatic approach in mathematics, Euclidean geometry follows from a set of given assumptions such as "all right angles are equal to each other"; Euclid originally had 5 postulates, 5 common notions and 23 definitions. To create broader geometries, one can systematically remove postulates to produce more general geometries with fewer restrictions.

It was the complementary transformation approach (Klein, 1957), however, that enabled mathematicians to first appreciate that Euclid's geometry was not the only geometry. In that approach, Euclidean geometry is characterized by whatever properties remain unchanged by a group of transformations known as isometries. Isometric transformations of a form allow the entire form as a whole to be relocated, but nothing else. All properties that remain unchanged-distance between any two points (length, size), angle formed by two lines, parallelism or non-parallelism on two lines, order of points along a line, are contained within the geometry. Position of a point, which is not invariant under isometric transformations, is not in the geometry. Klein showed how a set of broader geometries could be created by considering groups of increasingly more radical transformations that alter more than just position. The result are geometries that contain fewer properties than Euclidean geometry, e.g.. angle, parallelism, order-but not size, which now stands outside along with position. At the other extreme, topological transformations allow not only relocation of a form but enough massaging to turn a straight line on the form to a curved one.

Note that the geometries that are created in this way, or through the removal of axioms, are broader because fewer properties within a geometry imply that there are a greater number of forms that are equivalent to one another. For instance, in Euclidean geometry, a square and a square in a new position are considered the identical form, but a square and a small square are not, because size is a property in Euclidean geometry. In broader geometries, where size is not a property, a square and a small square are identical to each other, just as a square and a square in a new position are-size cannot distinguish between them, because size is not a property. Hence, a greater number of forms are all indistinguishable from one another. The more radical a transformation, the more properties that are altered, the fewer the properties that remain in the geometry and the larger/more general the resulting geometry. Table 1 shows are the ordered geometries that Klein demonstrated in a series of lectures now known as the Erlanger program, along with the properties within each geometry and the transformations that generate them (e.g., Modenov and Parkhomenko, 1965; Bedford, 2001).

Table 1: Family of geometries

\begin{tabular}{|c|c|c|c|}
\hline Geometry/ transformation & $\begin{array}{l}\text { Properties altered by the } \\
\text { transformation group }\end{array}$ & $\begin{array}{l}\text { Properties in the geometry } \\
\text { (invariant to transformation) }\end{array}$ & $\begin{array}{l}\text { Algebraic expression of } \\
\text { transformation group }\end{array}$ \\
\hline Euclidean/Isometric & Position & $\begin{array}{l}\text { Size*, angle, parallelism } \\
\text { collinearity, order, connectivity }\end{array}$ & $\begin{array}{l}X^{\prime}=a X+b Y+m(\text { where } \\
\left.Y^{\prime}=-b X+a Y+n \quad a 2+b 2=1\right)\end{array}$ \\
\hline Similarity & Position, size* & $\begin{array}{l}\text { Angle, parallelism, collinearity } \\
\text { order, connectivity }\end{array}$ & $\begin{array}{l}X^{\prime}=a X+b Y+m \\
Y^{\prime}=-b X+a Y+n\end{array}$ \\
\hline Affine & Position, size, angle & $\begin{array}{l}\text { Parallelism, collinearity, order, } \\
\text { connectivity }\end{array}$ & $\begin{array}{l}X^{\prime}=a X+b Y+m \\
Y^{\prime}=c X+d Y+n\end{array}$ \\
\hline Projective & $\begin{array}{l}\text { Position, size, angle, } \\
\text { parallelism }\end{array}$ & Collinearity, order, connectivity & $\begin{array}{l}X^{\prime}=(a 1 X+a 2 Y+a 3) /(c 1 X+c 2 Y+c 3) \\
Y^{\prime}=(b 1 X+b 2 Y+b 3) /(c 1 X+c 2 Y+c 3)\end{array}$ \\
\hline $\begin{array}{l}\text { Topology/topological } \\
\text { "Non-topology" }\end{array}$ & $\begin{array}{l}\text { Position, size, angle, } \\
\text { Position, size, angle, } \\
\text { parallelism, collinearity, } \\
\text { order, connectivity }\end{array}$ & Order, connectivity & $\mathrm{X}^{\prime}=\mathrm{g}(\mathrm{X}, \mathrm{Y})$ continuous functions $)$ \\
\hline
\end{tabular}

*: Preservation of the property "size" in Euclidean geometry is more precisely preservation of the distance between any pair of points, the strongest property that remains invariant in Euclidean geometry; altering "size" to yield Similarity geometry means allowing the distance between pairs of points to change, but preserving the ratio of distances between any pair of points 
I suggest for object identity that two samples of any sort, s1 and s2, can always be considered two forms. For each point on s1 there is a corresponding point on s2 to which it gets mapped by a transformation. One can identify the transformation and the level of geometry it produces. The more radical the transformation and the broader the level of geometry, the less likely those two samples will be judged to refer to one object. The "strongest" criteria, whereby two samples are judged very likely to be one object, corresponds to the smallest and richest geometry within which few distinct forms are considered identical. As the criteria get "weaker" and samples are judged increasing less likely to be one object, they correspond to increasingly larger geometries within which more and more distinct forms are considered identical. In this view, there is no single necessary and sufficient criterion of object identity, but a graded set of lesser and lesser preferences. In general, two samples that differ by an isometric transformation are the most preferred to refer to the same object. After that, two samples that differ by a similarity transformation is preferred. Similarity transformations will be more preferred for resolving identity than two samples that are related by an affine transformation, which is in turn is ranked higher than projective transformations. Projective transformations are more likely to lead to a same object conclusion than topological transformations, which finally are more likely than if properties of topology are violated as well. Most circumstances produce multiple samples. Thus, critically, identity is changeable and will be resolved in favor of the lowest level transformation available.

Geometry is a natural candidate for analysis of extended contours. An entire hierarchy of geometries rather than a single familiar geometry more accurately captures the vast range of possible object transformations all the way from simple position changes of Euclidean geometry to complicated plastic deformations permitted within topological geometry (Discussion). Geometric analysis of identity need not be restricted to Euclidean because it is the geometry human observers reason with consciously (and often badly) nor because it is believed to describe the local physical world we inhabit. No axioms believed false of our world are added to generate the geometries. Thus, the hierarchy is an evolutionary plausible solution as well as a powerful one (Shepard, 2001 for discussion of evolutionary constraints on perception). Moreover, great power is achieved simply by temporary removal of some of the axioms of Euclidean geometry (from the axiomatic perspective). Finally, it is abstract enough to be applied to all samples s1 and s2, in all the domains that object identity is required, including integration across space, time, sense modalities and left and right eyes. We believe it is the only possible candidate that provide a general core of identity.

\section{MATERIALS AND METHODS}

Experiment 1: The simplest prediction from the hierarchy for apparent motion is that object identity will be more likely when two stimuli have the identical form than when they have different forms. If a stimulus differs from an initial stimulus only in overall location and is the same in all other ways, then the two stimuli differ only by an isometric transformation. Informally, we think of this transformation as resulting in the "same form". According to the hierarchy, two stimuli that differ by an isometric transformation should be more likely judged to arise from the same object than two stimuli that differ by more radical transformations, such as topological transformations at the other extreme when squares can be turned into circles. Informally, we think of these transformations as producing "different forms".

Yet the role of form in apparent motion is controversial. As others have noted (e.g., Mack et al., 1989), some researchers argue that whether the form of the two stimuli are the same or not is irrelevant for apparent motion (Kolers and Pomerantz, 1971; Green, 1986; Kolers, 1972; Navon, 1976), whereas others argue the opposite (Orlansky, 1940). In addition, rigid motion, where all parts of a stimulus are seen to move by the same amount and the same rate, are important (Farrell and Shepard, 1981; Shepard, 1982; Shepard and Judd, 1976; Warren, 1977), but plastic formchanging deformations have been reported as well (Kolers and Pomerantz, 1971; Shepard and Judd, 1976; Farrell and Shepard, 1981). The purpose of the first experiment is to test the coarsest grain prediction from the geometric hierarchy theory of object identity: Is apparent motion more likely to occur between two stimuli that differ by a level 1 isometric transformation ("same form") than between two stimuli that differ by a level 5 topological transformation ("different form")?

The competing motion paradigm (Ullman, 1979) enables a direct comparison between levels of the hierarchy. At time 1, a stimulus appears in the center of the display and at time 2, two stimuli appear, one to the left and one to the right (Fig. 1). The direction of motion, either left or right, indicates which stimulus "wins". Because there is a choice between two stimuli, the selection of one over the other should indicate that this stimulus was determined to be more likely to come from the same object as the stimulus at time 1 . 


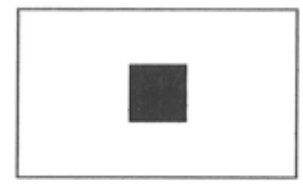

$t_{1}$ $t_{2}$

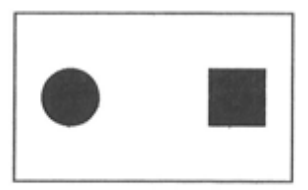

Fig. 1: Left-right competing motion paradigm. A figure appears briefly in the center at time $1(\mathrm{t} 1)$ and is followed a short time later ( $\mathrm{t} 2$ ) by two flanking stimuli. The original figure will be seen to move either left or right

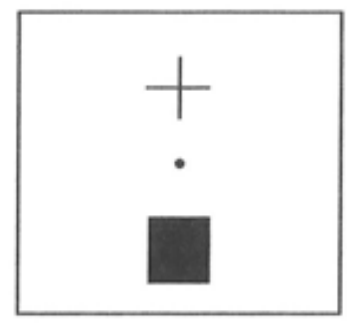

$t_{1}$

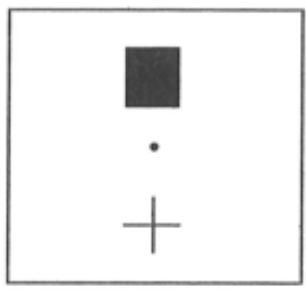

$t_{3}$

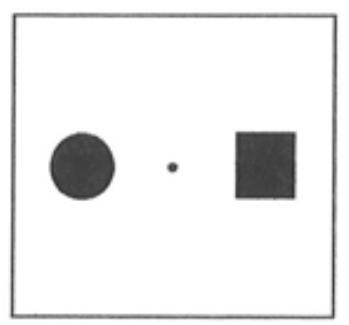

$\mathrm{t}_{2}$

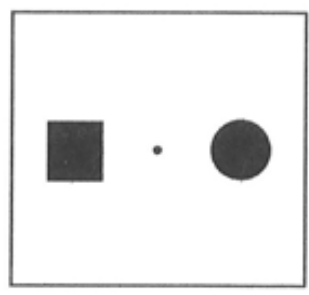

$\mathrm{t}_{4}$
Fig. 2: Clock paradigm. $\mathrm{T}=$ time. $\mathrm{t}_{1}$ : A figure appears on the bottom with a neutral plus on the top. $t_{2}$ : A choice between two figures, one on the left and one on the right. $t_{3}$ : The figure and the cross have switched places. $\mathrm{t}_{4}$ : The two choice figures have switched places. The original figure will appear to move clockwise or counterclockwise. The neutral cross will "soak up" the stimulus that is not chosen

Thus, the paradigm can be used to determine if a square (for instance) is more likely to "choose" the identical square over a circle (for instance) for resolving identity.

However, a practical problem with the paradigm is that observers can have an experience of "splitting" in which the unchosen stimulus also appears connected to the stimulus at time 1, although not as strongly (e.g., Dawson, 1989). The original stimulus appears to split, with a ghost of the stimulus appearing to weakly go to the other stimulus as well as the stimulus in the opposite direction. Such split experiences can undermine a subject's confidence in seeing a sensible single direction of motion. We believe this results from perceptual optimization of a situation that may force an unnatural 1-to-many mapping as the best of two poor non-preferred choices. To see a single clear direction would require seeing an odd-man-out stimulus appearing and disappearing in coincidental alternation with a moving object. To see two equally clear directions of motion would require one object turning into two, also not a natural resolution (In the geometric framework, such a transformation would be even more radical than a level 5 topological transformation and hence the least preferred (though not impossible) way to resolve identity. A transformation which splits one point into two destroys even the topological property of continuity and leaves little about the original form invariant). To produce a more stable situation, we devised a paradigm where an additional neutral stimulus is added in order to "soak up" as it were whichever stimulus is not chosen. The paradigm is shown in Fig. 2. At time 1 a form (e.g., square) appears at the bottom and the neutral stimulus (a "plus sign") appears at the top. At time 2, the two choices appear, one on the left (e.g., circle) and the other on the right (e.g., square). At time 3, the original two stimuli appear in reverse positions (e.g., square on top, neutral stimulus on the bottom). Finally, at time 4, the two choice stimuli appear in reverse positions and the sequence loops back to frame 1 . Note that if subjects determine that it is all the squares that refer to the same object, then a square will be perceived to undergo circular motion in a counter clockwise direction for the stimulus positions described above. Previous research has shown that apparent motion of a stimulus presented at the edges of a square will appear to smooth to a circle when the stimuli are presented quickly and repeatedly.

Both this clock paradigm and the original competing left-right motion paradigms will be used in the study. The clock paradigm is not perfect either-if identity is resolved by matching square to square, then the remaining stimulus will appear to unnaturally turn into a plus sign and turn back again. Consideration of the results of both paradigms together may offset any task-specific findings. To summarize, the prediction for Experiment 1 based on the theory is that when given a choice between a level 1 isometric transformation (i.e., "same form") and a level 5 topological transformation ("different form"), the isometric transformation will be selected.

\section{Methods:}

Subjects: The subjects were 10 undergraduate students ( 8 female, 2 male) at the University of Arizona who received course credit for participating. 
Stimuli: The stimuli were three filled geometric primitives, a square, a circle and a triangle. Four pairs of stimuli were generated, consisting of all permutations that use the square: Square-Circle (SC), Circle-Square (CS), square-triangle (ST) and TriangleSquare (TS). For type SC, the square is presented first, followed by a choice between the square and a circle. In type $\mathrm{C}-\mathrm{S}$, the circle is presented first, followed by a choice between the square and the circle. Likewise for the other two pairs. Three additional displays were generated consisting of all Squares (S), all Circles (C) and all Triangles $(\mathrm{T})$. The sides of the square were 15 $\mathrm{mm}$. The diameter of the circles was $15 \mathrm{~mm}$ and the width of the middle part of the triangle was $15 \mathrm{~mm}$. The four experimental stimuli are shown in Fig. 3.

\section{Paradigm:}

Clock: Each trial began with a fixation point in the center of the screen. Subjects were instructed to keep their eyes on the fixation point for the duration of the trial. When they were ready, they pushed a button on a computer mouse which began the trial. A trial consisted of 4 different frames cycled through repeatedly for at least $20 \mathrm{sec}$ (as discussed below). In the first frame, the initial stimulus (square, circle, or triangle) began in the position underneath the fixation point ("6 o'clock") and the neutral plus sign above ("12 o'clock"). The second frame presented the two competing stimuli (e.g., square and circle) to the left and right of fixation ("9 o'clock" and "3 o'clock"). The third frame displayed the same two stimuli as frame 1 but in reverse positions (12 and 6 o'clock swapped) and the fourth frame presented the same two stimuli as frame 2 in reverse positions. The distance between the fixation point and the edge of each figure was $14 \mathrm{~mm}$.

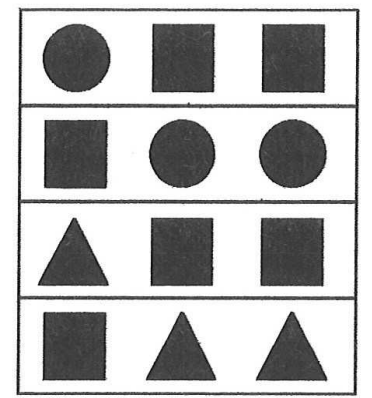

Fig. 3: Form conditions for Exp. 1. The stimuli are shown arranged for the left-right paradigm, if all 3 stimuli were visible at the same time. Top row shows condition S-C (square-circle); second row is $\mathrm{C}-\mathrm{S}$ (circle-square); third row is $\mathrm{S}-\mathrm{Q}$ (squaretriangle); fourth row is T-S (triangle-square)
Subjects were informed that after looking at a display for a while, they may notice that the "objects" appeared to be rotating clockwise or counter clockwise, which was demonstrated by the experimenter with a hand motion in the air. On each trial, a "settling in" period was allowed to accommodate the fact that not all subjects experience rotation early in viewing, especially for the first few trials. As soon as a subject noticed rotation, s/he informed the experimenter, who then set a timer for $20 \mathrm{sec}$. If a subject did not notify the experimenter within one minute, the trial was moved to the end of the block and the next trial was begun. For the timed $20 \mathrm{sec}$ interval, subjects were warned that the direction of motion could appear to switch while viewing the display. At the end of the interval, they were required to state verbally whether the direction of motion seen most often during that time was clockwise or counter clockwise and to rate the confidence in their judgment (0-guessing to 10-completely certain). The duration of each frame was $400 \mathrm{~m} \mathrm{sec}$ and the Inter Stimulus Interval (ISI) was $200 \mathrm{~m} \mathrm{sec}$.

Left-right: Each trial began with a fixation point in the middle of the screen. Subjects pushed the button on the mouse when they were ready for the display. A trial consisted of two frames which were cycled repeatedly for a fixed $6 \mathrm{sec}$. The first frame presented the initial stimulus in the middle of the screen, which replaced the fixation point. The second frame presented the two competing figures, one to the left and the other to the right of center. The distance between nearest edges of figures from frame 1 and 2 was $30 \mathrm{~mm}$. Subjects were required at the end of the $6 \mathrm{sec}$ to indicate if the direction of motion they saw most often was "left" or "right" and to rate confidence in their judgment.

Procedure: The subject was seated $50 \mathrm{~cm}$ from the computer monitor and the height of a chair adjusted such that eye level matched the center of the screen. Displays were created and shown using Animation Works software and run on an IBM computer. Subjects were given 2 blocks of each paradigm, presented in alternation: Clock, left-right, clock, left-right. A block consisted of 11 trials, 2 repetitions of each of the 4 conditions (S-C, C-S, S-T, T-S) and 1 repetition of the ambiguous controls (S,C,T). Direction of motion was counterbalanced such that the lower level transformation ("same form") was in one direction for one of the repetitions (clockwise or left) and the other repetition in the other direction (counter clockwise or right). Trials were presented in random order. Each subject received a total of 44 trials for a session that lasted approximately $50 \mathrm{~min}$. 
Current Research in Psychology 1 (1): 35-52, 2010

\section{RESULTS}

The stimuli were perceived to move to the same form nearly $70 \%$ of the time (mean $=69.7 \mathrm{t}=3.56$, $\mathrm{p}<0.005)$ overall, $75.6 \%$ of the time $(\mathrm{t}=3.99$, $\mathrm{p}<0.0025)$ in the left-right paradigm and $63.7 \%$ $(t(9)=2.47, \mathrm{p}<0.025)$ in the clock paradigm. All are greater than chance, which would be $50 \%$ (The data exclude the three ambiguous control conditions, where all three forms are identical, so as not to artificially inflate any motion to "same form" result. Means and errors for the four experimental conditions for all $t$ tests are calculated on 1 mean score for each subject for the relevant condition so as not to artificially decrease the error term).

The data are shown separately for each of the four (experimental) form conditions in Fig. 4. As can be seen in the Fig. 4, the probability of motion to the square changes as function of what the initial form is; for the Square-Circle and Circle-Square pairs, motion to the square is greater when the initial form is a square (S-C) than when it is a circle (C-S) and for the squaretriangle and triangle -square pairs, motion is greater to the square when the initial form is a square (S-T) than when it is a triangle (T-S). Difference between form conditions was confirmed by an ANOVA (although the data are categorical, an ANOVA is preferable to a chisquare analysis. A chi-square assumes the each data point is independent (different subjects). Since the data are not, a large difference in only 1 or 2 subjects could produce significant results for the whole group. In addition, summary statistics, such as the mean, are meaningful for these data, unlike some types of categorical data) performed on the data (probability of motion to square) with form condition (S-C, C-S, S-T, $\mathrm{T}-\mathrm{S}$, paradigm (left-right, clock) and block $(1,2)$ as factors, where the main effect of form condition proved significant $(\mathrm{F}(3,27)=11.26, \mathrm{p}<0.001)$. No other main effect or interaction was significant.

To analyze the data more closely, an ANOVA was also performed on probabilities of motion to the same form, where main effects of paradigm $(\mathrm{F}(1,9)=8.38$, $\mathrm{p}<0.05)$ and form condition $(\mathrm{F}(3,27)=5.06, \mathrm{p}<0.01)$ were significant. The paradigm effect reflects the greater overall probability of same-form motion for the left-right paradigm than for the clock paradigm, although both are significant as noted above. The form condition effect here reflects a greater same-form effect for some stimuli over others (means: S-C, 75\%; C-S; $71.2 \%$; S-T, 56.2\%; T-S, 76.2\%). That is, when the distracter item was a triangle, the initial form (square) did not go the same form as often as the other conditions. One possibility is that triangles tend to be perceived as pointing in a particular direction and may have commanded attention.
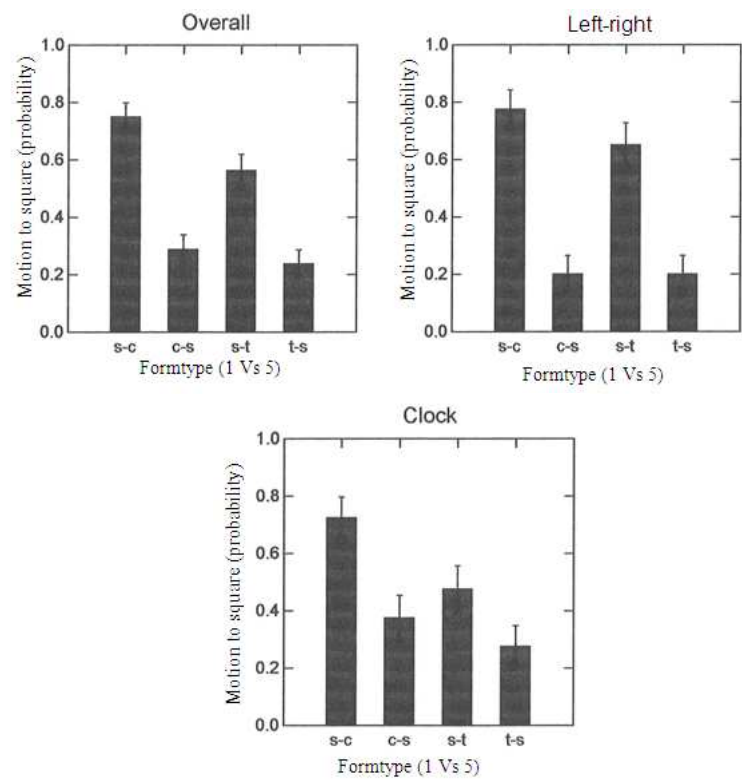

Fig. 4: Data for Exp. 1. Probability of motion to square as a function of the four experimental form conditions both overall and for each paradigm (clock and left-right) separately. $\mathrm{S}=$ Square, $\mathrm{c}$ $=$ Circle, $\mathrm{t}=$ Triangle. Note: all four conditions reflect a level 1 Vs 5 choice. Error bar is 1 standard error of the mean

To check for direction preferences, separate ANOVAs were performed on each paradigm to include motion direction as a meaningful factor (clockwise and counter clockwise for the clock paradigm and left and right for the left-right paradigm). There were no direction preferences for the clock paradigm. In the other paradigm, there was a marginally significant $(\mathrm{F}(3,27)=2.60 \mathrm{p}=0.073)$ direction by form condition interaction. This reflects a complicated tendency to map to the square when the square was on the right rather than the left for the two form conditions containing the circle and the square, but a tendency to map away from the square when it was on the right rather than the left for the two form conditions containing the triangle and the square. If this is a real effect, it could be that mapping to the "right" position in the left-right paradigm holds a special preferred status and in addition, a square is a preferred target over a circle and a triangle over a square, independent of the originating stimulus. This interpretation would also be consistent with the differences between the form conditions discussed above.

Finally, the confidence ratings were higher in the clock paradigm (8.2 out of 10) than in the left-right paradigm (6.6), even though the probability of 
matching to the same form was higher in the left-right paradigm. The difference was verified by an ANOVA on confidences with paradigm, form condition type, block and direction as factors, where the main effect of paradigm was significant $(F(1,9)=6.37, \mathrm{p}<0.05)$ and nothing else. Thus, subjects were less certain of the motion direction in the left-right paradigm suggesting that some splitting of the original form may have occurred.

The findings confirm the simplest prediction of the geometric theory of object identity: Form matters. When observers have a choice between a figure that differs from the initial form by an isometric transformation and a figure that differs by a topological transformation, identity is resolved in favor of the lower isometric level. This finding occurred even in a paradigm (clock) that produces high confidence in what is seen and is not very transparent about what ought to be seen based on expectation or deliberate problem solving. Perceived direction of apparent motion is not random but is more likely to occur towards the "same stimulus" than towards a "different stimulus". The results are consistent with investigators who argue that apparent motion is sensitive to a changes in form. Claims to the contrary will be considered in the general discussion.

Experiment 2: The next step was to examine what would happen when neither choice was identical to the figure seen a fraction of a second earlier. That is, if a level 1 isometric transformation is not one of the choices for resolving identity, what will happen to apparent motion? Numerous studies report that apparent motion does not break down when two stimuli differ in some way other than location. For instance, a square will appear to deform and reform into a circle as it moves from one side of a display to the other. If there is a choice between two non-identical stimuli, will the direction of motion be random, or is there some basis from which to choose? The specific prediction of the geometric theory is that whichever option is related to the original stimulus by a transformation from a lower level of the hierarchy will be judged to refer to the same object and apparent motion will be experienced to occur between that pair.

Consequently, Experiment 2 tested a finer-grain prediction of the hierarchy than Experiment 1 by moving up a level to level 2, similarity transformations, where neither of the choices could informally be "same stimulus". Similarity transformations are slightly more radical than isometric transformations and allow not only equal changes to the locations of all points, but also unequal changes to locations, provided all lengths are changed uniformly. That is, permitted transformations are those we regard informally as changes in "size" (Table 1). The level 2 transformation will be pitted against the other geometric extreme, a topological transformation, as in experiment 1 . The experiment will determine if a square is more likely to considered the same object as a square of a different size or as a circle (level 2 Vs 5). The outcome will be compared to a condition where a square has the option of moving to a square of a different size or to an identical square (level $1 \mathrm{Vs} 2$ ). The theory predicts that motion to the different size square will be greater for the former pair of choices, then for the latter, where it should move away from the different-sized square. The remaining permutation (level 1 Vs 5) completes the triad and a fourth control condition where it was expected motion would be equally likely in both directions was included, as in the first experiment. The stimulus conditions are shown in Fig. 5.

\section{Method:}

Subjects: Ten different undergraduate students from the University of Arizona served as subjects. They received course credit for their participation.

Stimuli: The square and the circle were identical to those used Exp. 1. A different-sized square was created by reducing the original square to $11 \mathrm{~mm}$ on each side, approximately $75 \%$ the size of the larger square. The three experimental conditions all began with the original square, followed by a choice between the original square and a circle ( 1 Vs 5$)$, between the original square and the smaller square (1 Vs 2) and between the small square and the circle (2 Vs 5). A fourth control condition consisted of the original square, followed by a choice between 2 triangles from Experiment 1, one pointing up and the other down (Control).

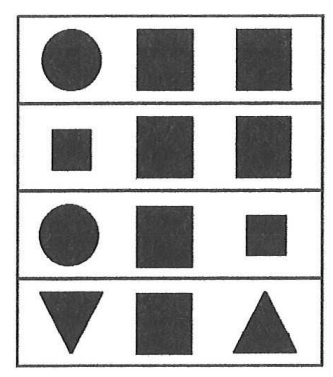

Fig. 5: Stimuli for Exp 2. Row 1: Level 1 Vs 5 (squarecircle); Row 2: level 1 Vs 2 (square-small square); Row 3: Level 2 Vs 5 (small squarecircle); Row 4: triangle control 

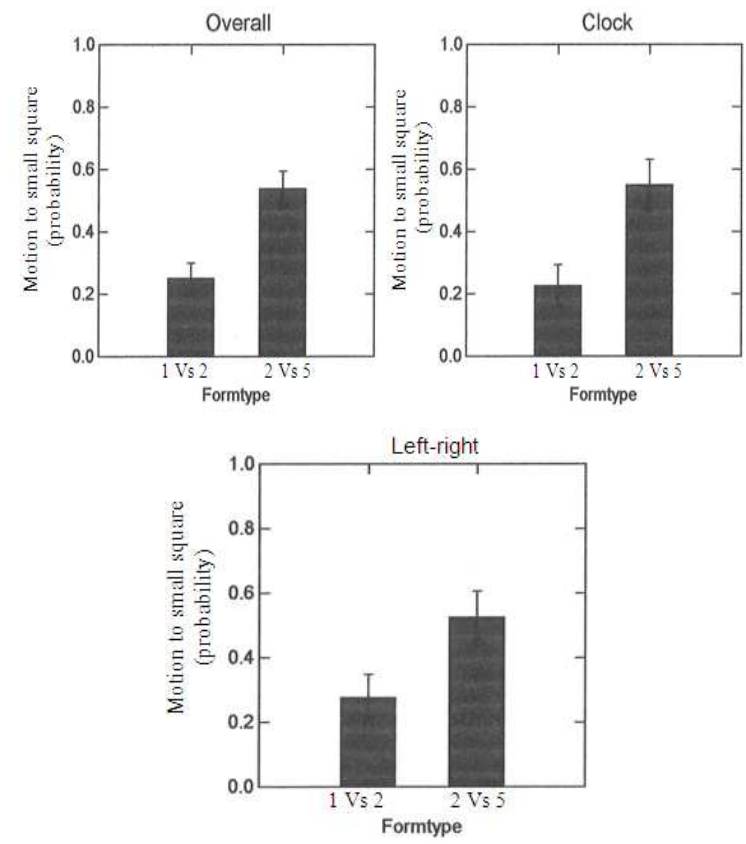

Fig. 6: Data for Exp. 2. Probability of motion to small square. Level 1 Vs 2 is the square vs. small square condition; level 2 Vs 5 is small square Vs circle condition

Paradigm and procedure: The clock and left-right paradigms were identical to Exp. 1. Also as in Exp. 1, there were 4 blocks of trials, in the order Clock, L-R, Clock, L-R. Each block consisted of 2 repetitions of each of the 4 form conditions, for a total of 32 trials per subject.

For condition 1-5, square Vs circle, the original square moved in the direction of the square rather than the circle $75 \%$ of the time overall $(\mathrm{t}=3.68, \mathrm{p}<0.001)$, $72.5 \%(\mathrm{t}=2.08, \mathrm{p}<0.05)$ of the time in the left-right paradigm and $77.5 \%(\mathrm{t}=3.16, \mathrm{p}<0.01)$ in the clock paradigm. This result replicates the "same form" result from experiment 1, where the square vs. circle condition was also used and where there was also an overall $75.0 \%$ motion to the square for that condition. For condition control, there was a tendency $(60 \%)$ for the square to move to the triangle with the point on top for the left-right paradigm, but the opposite tendency in the clock paradigm, where the square moved to the triangle with the point on top only $42.5 \%$ of the time. Neither value reached significance $(t=1.18, p>0.1 ; t=$ $1.15, \mathrm{p}>0.1)$. It was expected that motion to each triangle would occur with equal probability in both paradigms, but this tendency to deviate is of no particular significance for the hypothesis under consideration; as noted in Experiment 1, triangles that appear to point in a direction may exert their own effect independent of the form effects under exploration here.

The two conditions of primary interest are shown in Fig. 6, which shows the proportion of trials the square moved to the small square for each paradigm. For clock, the square moved to the small square only $22.5 \%$ in condition $1-2$, but did so more than twice as often at $55 \%$ in condition 2-5 (difference $\mathrm{t}=3.28$, $\mathrm{p}<0.01)$. Similarly for left-right, $27.5 \%$ motion to the small square in condition 1-2 increased to $52.5 \%$ $(\mathrm{t}=1.79 ; \mathrm{p}<0.10)$ in condition $2-5$. Likelihood of motion between the same exact two stimuli, a square and a small square, was pushed around by the other stimuli present in the situation. That is, whether or not two stimuli will be determined to refer to the same object is not fixed, but is a function of the choices available in the situation. The direction of the results are consistent with the prediction of the hierarchy: The square is more likely judged as the same object as the small square if the only other choice is from a higher level of the hierarchy (level 5 circle), than if the other choice is from a lower level of the hierarchy (level 1 square).

However, a less interesting interpretation is that the data only appear to be consistent with levels of a hierarchical structure. Inspection of just condition 2-5 by itself does not show any clear tendency for the square to move to the small square rather than the circle, as would be also predicted by geometry. Perhaps there is only a tendency to match to the identical stimulus, as shown in experiment 1 (with only minimal processing of the non-identical stimulus). This would account for motion of the square to the same square rather than the small square in condition 1-5, but once the identical square is removed (condition 2-5), identity and hence direction of motion, would be at chance. That is, perhaps there are no multiple hierarchical levels. Alternatively, the low absolute probability of moving to the small square rather than the circle could result from a confound involving distance. Only smaller squares and not larger ones, were used in this study and placed such that the midpoint was in the same position as the midpoint of the larger figures. This creates a display in which the nearest edge of the small square is further from the original square than the other choice, the circle. The nearer stimulus may have exerted its own pull, in a direction opposite of any tendency to move towards the small square. What appears as random selection between two stimuli could be the result of competing tendencies that counteract one another. While distance reflects only an "innocuous" isometric transformation, the choices must nonetheless be equated for amount of transformation for a "fair" 
comparison of the levels. Distance between stimuli is known to affect apparent motion; whether it did so here is unclear.

Remaining analyses using an ANOVA on probability of motion to a "lower level" finds no difference in this experiment between clock and leftright paradigm (considered both with and without the control group), no direction preferences and no tendency for differences between block 1 and 2. There was a significant difference between the form condition type $(\mathrm{F}(3,27)=4.74, \mathrm{p}<0.01)$ reflecting both the Control data and the $50 \%$ performance in condition 2-5 as discussed above. Finally, subjects were again more confident of their judgments in the clock paradigm, with a mean rating of 6.7 out of a possible 10 for clock and 4.6 for left-right. An ANOVA on confidences finds this main effect of paradigm significant $(\mathrm{F}(1,9)=14.53$, $\mathrm{p}<0.005$ ), as well as a significant main effect of form condition type $(\mathrm{F}, 3,27)=8.59, \mathrm{p}<0.001)$ and $\mathrm{a}$ paradigm by block interaction $(F(1,9)=9.84, p<0.025)$. The form effect reflects least confidence about what seen in the control condition (4.8); the other 3 were higher and more similar to one another (5.6, 6.3, 5.8 for $1 \mathrm{Vs} 2$, 1 Vs 5,2 Vs 5 respectively). The interaction reflects a tendency for subjects to get more confident by block 2 for the left-right paradigm whereas confidence in the clock paradigm was steady.

Experiment 3: The purpose of Experiment 3 was to disentangle any effect of form from other confounding factors, such as distance. The conditions of the last experiment were repeated, except with each small square replaced by a small circle (Fig. 7). In the previous experiment, when there was a choice between a small square and a circle, the square was seen to move to each stimulus equally often. By replacing the small square with a small circle, any pull from the squareness is removed; both choices are circles. That is, both choices are now level 5 topological transformations of the original square. Any tendency to move to the closer figure should now be unopposed. If the equal motion to the two stimuli observed in the previous experiment was due to two tendencies that counteracted one another, then the square in this experiment should be seen to move to the closer circle. If on the other hand the different distances involved were too minimal to have exerted any influence and chance performance was just that, then the square should be seen here to move to the circle and the small circle equally often.

\section{Method:}

Subjects: The subjects were 10 different undergraduates at the University of Arizona who received course credit for participating.

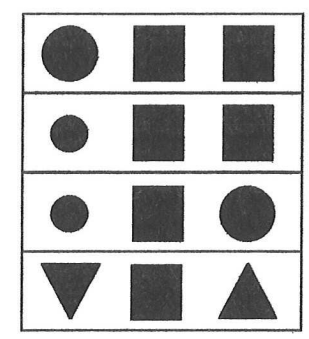

Fig. 7: Stimuli for Exp. 3. Row 1: Level 1 Vs 5 (square-circle); Row 2: Level 1 Vs 5s (squaresmall circle); Row 3: Level 5 Vs 5s (circle-small circle); Row 4: triangle control

Stimuli, paradigm and procedure: These were identical to experiment 1 , except as noted above, each small square was replaced with a small circle. The diameter of the small circle was the same as the length of the sides of the small square. The fourth "control" condition using the triangles was also included to maximize the similarity between the experiments. General piloting suggested that types of displays on other trials influence performance.

The control triangle condition showed the same tendency as Experiment 2 for the square to map onto the triangle with the point on top in the left-right paradigm $(57.5 \%)$, not in the clock paradigm $(35 \%)$ (difference marginally significant, $t=1.96, \mathrm{p}<0.10$ ). Condition 1-5, (large) square vs. (large) circle again shows greater than chance motion to the square overall $(81.2 \%, \mathrm{t}=7.11, \mathrm{p}<0.001)$ and for both left-right $(90 \%, \mathrm{t}$ $=7.24, \mathrm{p}<0.001)$ and clock $(72.5 \%, \mathrm{t}=3.86, \mathrm{p}<0.005)$ paradigms, as in Experiments 1 and 2. In condition 1$5 \mathrm{~s}$, (large) square vs. (small) circle, the square was chosen over the circle $85 \%$ of the time for left-right and $75 \%$ for clock $(\mathrm{t}=3.87, \mathrm{p}<0.005)$. This is expected both from the "same form"/lower level effect, as well as from any tendency to move to the closer figure.

The data for these two experimental conditions, as well as the third experimental condition, are shown in Fig. 8. In the condition of greatest interest, $5-5 \mathrm{~s}$, when there was a choice between the closer circle and the further smaller circle, the square did indeed move to the closer circle $77.7 \%$ of the time (75\% in left-right, $80 \%$ in Clock), substantially more often than would be expected based on chance $(\mathrm{t}=5.77, \mathrm{p}<0.001 ; \mathrm{t}=3.35$, $\mathrm{p}<0.005 ; \mathrm{t}=4.81, \mathrm{p}<0.001$ respectively). The data show that there is a pull from the closer figure, unmasked here where both choices are circles. We conclude that seeming chance performance in condition $2-5$ in the previous experiment was indeed due to two counteracting forces. Were there no attraction from the small square (level2), the large square would have 

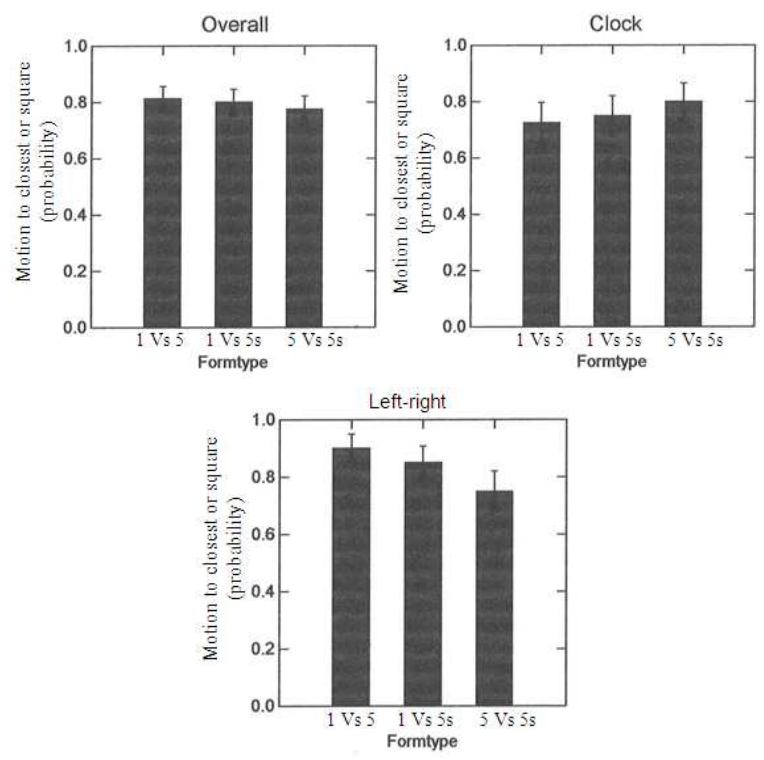

Fig. 8: Data for Exp. 3. Probability of motion to the closest figure, or to the square for condition $1 \mathrm{Vs}$ 5

moved to the closer large circle $77 \%$ of the time, as it did here, rather than only $47 \%$. For the square to have moved to the further figure as much as it did , 53\%, there must have been an another influence.

Remaining findings based on ANOVAs conducted on probability of motion to the closest figure (or to the square for the 1-5, square-circle condition) show a difference between form conditions $(\mathrm{F}(3,27)=15.52$, $\mathrm{p}<0.001)$, which is due to only the control condition. Differences in paradigm without the control condition were not significant. In the left-right paradigm, there is a direction main effect; the square was more likely to move to the closer circle when the closer circle was on the right $(83.7 \%)$ than when it was on the left $(70.0 \%)$ $(\mathrm{F}, 1,9=6,44, \mathrm{p}<0.05)$. This is not unlike the marginally significant tendency in experiment 1 for the position on the right to be preferred. For the clock paradigm, there was also a direction effect, with a more complicated triple interaction of condition by direction by block. It is possible that when solutions based on form are not possible, direction biases are more likely to manifest themselves.

Confidences are again higher for clock (7.6) than for left-right $(6.2), F(1,9)=8.48, \mathrm{p}<0.025$. The only other significant effect on confidences is for form condition $(F(3,27)=18.69, p<0.001)$, which again finds the ambiguous triangle control rated substantially less confident (5.7) than the other three conditions (circle Vs. square $=7.3$; circle vs. small square7.6; circle Vs small circle 6.9). Note that for the three experimental conditions, the confidence tends to be lowest when both choices are less preferred topological transformations, even though one circle is closest.

The results of Experiment 2 and 3 taken together suggest that when the "same stimulus" is not an available choice, there are nonetheless rules for achieving object identity and apparent motion. Motion direction is not chosen at random. When distances between nearest edges are equated, there is a clear preference to move from a square to a small square, rather than a circle. In the geometric framework, a square is a more radical transformation (level 5) than a transformation which changes scale and nothing else (level 2) and identity favors the lower level. And as noted earlier, how identity is achieved depends on the choices in the situation. As with findings in the literature concerning form, these results seem consistent with reports that the property of size is relevant in apparent motion (e.g., Burt and Sperling, 1981) and contrary to those that argue the opposite (e.g., Mack et al., 1986; Navon, 1976). This contradiction will likewise be revisited in the general discussion.

A theoretical question of note: Isn't intuition just as useful as the geometric hierarchy? Contrary reports on form and size not withstanding, isn't is simply intuitively obvious that a square and a small square are more "similar" than a square and a circle and motion will simply be seen between the similar pair? What the geometric hierarchy does is to formalize and make more rigorous what is meant by "similar" in this context. If it often corresponds to intuition, so much the better.

Experiment 4: The final experiment sought to determine if additional lesser criteria of object identity could be coaxed to reveal themselves. Thus far, evidence for two preference levels has been found: A primary preference for resolving identity using a stimulus differing only in location (level 1), over a radical deformation (level 5) and a secondary preference when level 1 is not an option to choose a smaller otherwise identical stimulus (level 2) over the radical level 5 deformation. It could be argued that genuine hierarchical structure requires demonstration of at least three distinct levels of preference. According to the geometric hierarchy, even the radical level 5 deformation ought to be identified as the same object as the initial figure if it is the lowest level choice in the situation.

When an identical square is unavailable, a square is pulled towards a smaller square rather than a circle(level 2 Vs 5), with distance controlled. The 
geometric hierarchy predicts that motion to the circle should be increased if the option of a small square is no longer available, but is replaced with an even more radical transformation than the circle, a transformation that does not even preserve topological properties such as order, connectivity and so on (level 5 Vs 6). Level 2 Vs 6 completes the triad.

\section{Method:}

Subjects: The subjects were 10 additional undergraduates at the University of Arizona that received course credit for their participation.

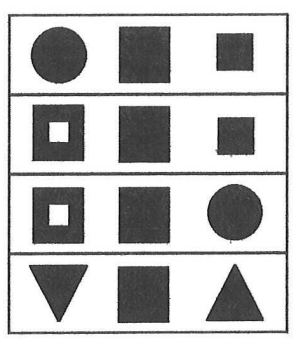

Fig. 9: Stimuli for Exp. 4. Row 1: Level 2 Vs 5 (small square-circle); Row 2: Level 2 Vs "6" (small square-square with hole); Row 3: Level 5 Vs "6"; circle-small square with hole. Row 4: triangle control
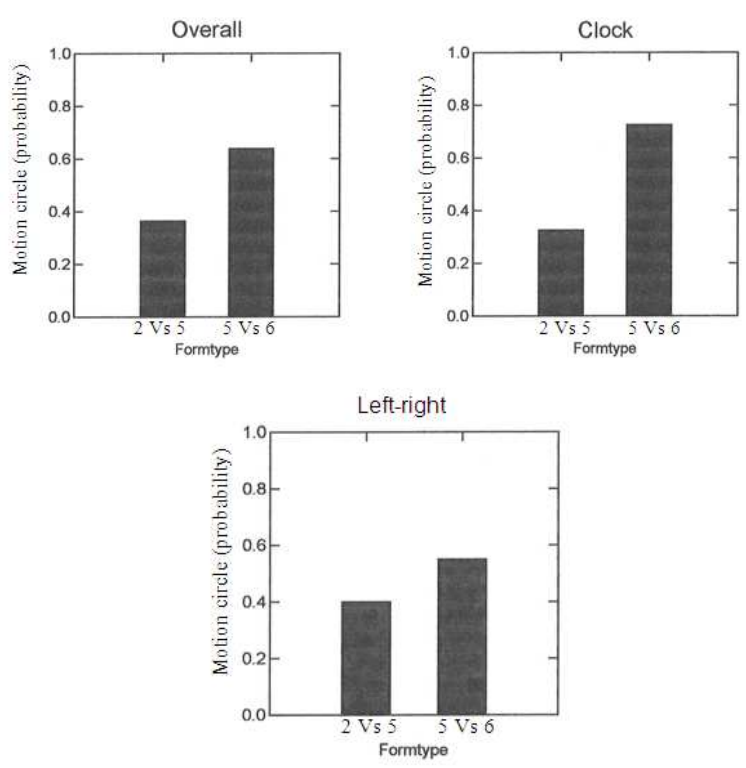

Fig. 10: Data for Exp. 4. Probability of motion to the circle. Condition $2 \mathrm{Vs} 5$ is small square Vs circle; $5 \mathrm{Vs}$ "6" is the circle vs. square with hole condition
Stimuli, paradigm and procedure: The original square, small square and circle were identical to previous experiments. For the additional level " 6 " stimulus, we used a stimulus after Chen (1985), a square with part of the interior missing. The "hole" extended $8 \mathrm{~mm}$ of the $15 \mathrm{~mm}$ sides of the square. A square and a square with a hole in it are not related topologically and are thus more dissimilar to one another geometrically than the square and the circle, despite some intuitions to the contrary. The three experimental conditions, $2 \mathrm{Vs} 5$, small square vs. circle, 2 Vs 6, small square vs. square with a hole and 5 Vs 6, circle Vs circle with a hole are shown in Fig. 9, along with the triangle control. All other procedural details are identical to previous experiments.

Probability of motion to the circle for Condition 25, small square vs. circle and Condition 5-6, circle vs. square with a hole, are shown in Fig. 10. The data suggest that identity resolution with the circle can in fact be made higher when the only other alternative is a more radical transformation. For the clock paradigm, when the other choice is a small square, the original square maps onto the circle $32.5 \%$ of the time, but increases to $72.5 \%$ when the other choice is a square with a hole in it (difference $t=4.71, p<.005)$. The effect is much less pronounced for the left-right paradigm, with an increase from $40 \%$ to $55 \%$, which did not reach significance, but nonetheless, overall, a square can be made more likely to map onto a circle, perhaps in some paradigms more than others.

Chen (1985) was the first to show that a square is more likely to move to a circle, than to a square with a hole in it. However, his exclusive use of the left-right competing paradigm called his results into question (Dawson, 1989) and his (chi-square) statistics make it possible that the effect was carried by a single subject. Dawson was not able to replicate his result for direction of motion, but was able to find an effect for goodness of motion. We find the results for direction of motion here more strongly using the clock paradigm, a paradigm that is much less open to influence of expectation and problem solving strategies and indeed has higher confidences in what is actually seen. The square with a hole in it may be a particularly stringent test of topology vs. non-topology, especially in the left-right paradigm. The stimulus may encourage splitting of the original square into the outside of the square (square with a hole) and an inside piece, rather than choosing one stimulus over the other. This would be less likely in the clock paradigm, where the presence of a fourth stimulus makes splitting less likely. A square with a hole in it could also be mistaken for a square with a pattern which would cause some pull from the stimulus 
since this would render it a geometrically innocuous transformation.

While additional stimuli are needed, the experiment nonetheless shows a square can be made to map to a circle if the alternative is less preferred. Note that the present results of square to circle rather than square with a hole in it also cannot be explained by any simple tendency to match figures based on their area. Even though the square and the circle were more similar in area than the square and the square with a hole in it, ty are also more similar in area than the square and the small square from condition 2-5, yet in this condition, the circle was chosen less often. The present experiment also shows when such square-circle motions will occur: If there is a small square present, motion to the circle is less likely. That is, a square will move to a circle if there is no lower-level, less radical, option. The present reearch grounds sensitivity to topological properties (Chen, 1985) in a larger hierarchical framework.

These data are consistent with frequent reports that plastic apparent motion between dissimilar forms such as a square and a circle can occur and provides a basis for understanding when such deformations will occur. We presume observers experienced a square deforming into a circle when it moved in that direction, but did not explicitly test for it.

Note that in condition 2-5, small square vs. circle, the probability of motion to the small square is $67.5 \%$ in the clock paradigm (60\% for left-right). This is greater than chance, $\mathrm{t}=3.28, \mathrm{p}<0.005$, even without a control for distance and higher than the probability of the same condition in Experiment 2. One possible contributing factor to this difference between experiments is that in this experiment none of the conditions had a level 1 or "same form" option, unlike all the previous experiments. As noted earlier, piloting suggested that cross-trial influences can occur. The lack of the most preferred choice in all of the trials may have made lesser preferences even stronger and more easily manifested. Condition 2-6 small square vs. square with a hole in it also was more likely than chance to move to the small square $(63 \%$ for Clock; $\mathrm{t}=1.46, \mathrm{p}<0.10)$, despite the fact that it was further away.

Subjects were more confident in their judgments for the clock paradigm than for the left-right paradigm (means=6.8 and 4.7; ANOVA main effect for paradigm, $F(1,9)=12.72, \mathrm{p}<0.01)$ a very consistent finding across all experiments in this study. The only other significant effect in the ANOVA is a form condition effect $(\mathrm{F}(3,27)=16.17, \mathrm{p}<0.001)$ which also like the other studies, reflects least confidence in the ambiguous triangle control condition (4.8 Vs 6.0-6.1 for the 3 experimental conditions).

\section{DISCUSSION}

The current set of studies shows multiple levels of a hierarchical structure for object identity. Whether or not two samples are determined to refer to the same object is not fixed, but depends on the choices available in the situation. In addition, resolution among choices shows transitivity. The ordered set of preferences for which stimulus refers to the same object as a square are as follows:

- If a choice between square and small square, choose square

- If a choice between small square and circle, choose small square

- If a choice between circle and a square with a hole, choose circle

- (Transitivity: If a choice between square and circle, choose square)

\section{Or generally:}

- if isometric or similarity transformation, choose isometric

- if similarity or topological transformation, choose similarity

- if topological or non-topological transformation, choose topological

- (Transitivity: If a choice between isometric and topological, choose isometric)

For apparent motion, the findings resolve some long-standing apparent contradictions in the literature. Investigators that come to different conclusions about the role of form are often asking different questions: Can changing the shape of the stimulus be shown to have an effect on apparent motion? The answer is "yes", shape matters. The second question: Can apparent motion occur even when the shapes are made different? The answer is also "yes", shape doesn't matter. They are both correct. The existence of multiple levels of criteria of object identity implies that apparent motion can be experienced when the shapes are radically different, while at the same time showing a preference for the identical shape if available. Shepard (1984; 2001) has argued that apparent motion shows a preference for rigid motion - in the current framework an isometric transformation-and suggests that when rigid motion is not possible, the system may use lesser 
criteria of object identity. The current research shows that there are lesser criteria and suggests what they are. They come from a hierarchy of nested geometries, of which isometric is the smallest. Contradictory beliefs about size similarly reflect different questions. In addition, there is some confusion over what it means to say there is an "effect of size" in apparent motion. It can refer to motion between two figures of different size and same shape but also to motion between two figures of the same size and different shape. The current set of studies is the first to demonstrate the changeablitity of apparent motion with respect to form, within a single paradigm and often within the same subjects and in a systematic rule governed way.

For object identity generally, the dynamic consequences of the hierarchical structure is precisely what is needed to explain how decisions can change across a wide range of situations. That is, a pair of samples with the very same geometric properties can sometimes refer to the same object, but other times not. A circle and an ellipse are related by an affine transformation. The two samples can refer to different objects, the circle a soccer ball, the ellipse a balloon. But they can also refer to the identical object as with a circular sponge molded into an elliptical shape, or a penny seen head on and again at an angle. A rectangle and a trapezoid will sometimes refer to a single door and other times to two distinct objects. Observers must make the correct judgment - sometimes yes, sometimes no- in all the varied circumstances if they are to perceive the world accurately and coherently. In the current view, changeability does not reflect chaos (Appreciation for the changeability may also put to rest endless debate in vision literature about whether various detection abilities are or are not position invariant, or are or are not size invariant and so on. Both sides are correct). Nor does it imply that different rules for different classes of objects need be relied upon to get different outcomes. The decision that is reached depends on the choices available in the situation. A sample will be matched to whichever sample is the least transformed, where transformed is defined by lowest level of the geometric hierarchy.

As noted earlier, the geometric hierarchy may capture intuitions about the "similarity" (for discussions of the concept "similarity" (Medin et al., 1993) of two items for the purposes of object identity. The more similar two samples, the more likely they will be judged as the same object. Yet intuitions can be misleading, geometry is more trustworthy. For instance, consider the use of "size" and its relation to shape and form. Some researchers both in development (Wilcox, 1997) and adult research (Goodale et al., 1991) regard size as a property that constitutes shape, in the temporal stream system that determines "what" is an object. This seems to match intuition-if a couch becomes smaller, it is not regarded as the identical object, whereas the couch can be reoriented and still be the same couch. Thus, size seems more like shape properties that constitute what an object is, such as angle and unlike irrelevant properties such as position and orientation. On the other hand, the opposite intuition is that if a square is changed to a small square, the "squareness" is somehow still there, unlike angle and more like position and orientation. Some researchers (Narter, 1998) seem to have this intuition instead and have argued that size may be more appropriately thought of not in a "what" system, but in a "where" system, of any what-where division of labor. Depending on intuition, size is placed in one of two very different categories.

In the present view, size (or any property) should not be placed in either category. Instead, the properties that constitute shape changes. As the geometry changes, so too will the role of size. Size will be a property of shape in Euclidean geometry, but will not be a property of shape in Similarity, Affine, Projective, or Topological geometry. Size can distinguish between forms in Euclidean geometry, but not in the broader geometries. And, as seen, the geometry that governs identity changes. Two samples that differ in size can be judged to refer to different objects, in which case we are governed by Euclidean geometry. Two samples can also be judged as the same object, in which case we are governed by Similarity (or broader) geometry. Wavering intuition could reflect some appreciation that geometry changes, but without the structure of the hierarchy, intuition can be misleading. Properties such as size are placed in one fixed category or another, depending upon relative strength of individual intuition.

The strict division between "what" Vs "where" systems itself (Mishkin et al., 1983) may be based largely on preconceived intuitions that Euclidean geometry is special, as much as it is on data. Position-or "where"-is the only property not in Euclidean geometry; all other properties are in the geometry. This may lead to an intuition of a hard division between position and all other properties. Yet the separate ventral and dorsal streams used to support what vs. where can also be interpreted differently if different intuitions are brought to bear, such as a "what" Vs "how" (Goodale and Milner, 1992), or a "point source" Vs "extended contour division" (Held, 1970; Bedford, 2003) division. In the present view, there is no hard and fast division between position and other geometric properties, despite the overwhelming influence that separate "what" and "where" systems have had in perception, cognition and development. 
Language is also biased by predominately Euclidean intuitions-at least the English language. Knowledge that there is a whole range of geometries that observers can exploit may make more precise terms such as "same form", "different form", "same stimulus", "shape" and "transformation". "Same form/stimulus/shape" usually means two figures that are identical in all ways except position and orientationthey can be brought into point for point correspondence by rotation and translation. If you cannot effectively pick one up and reposition it such that it would lie perfectly on the other, then it is a different stimulus. Yet this only holds true for Euclidean Geometry. In the present view, two stimuli cannot be determined to have the "same form" or not, until one specifies the geometry. A more precise general definition is warranted, such as: Two shapes are the same if and only if one can be brought into point-for-point correspondence with the other by any transformation in the group of transformations that defines the relevant geometry.

Why does the family of geometries work for object identity? They may mirror the wide range of transformations that objects produce at the level of the proximal stimulus and beyond. In Table 2 there are different transformations that objects produce that correspond to different levels of the hierarchy. The collection is on-going, especially for affine and projective transformations of objects themselves. Note that while this makes the geometric transformations ecologically valid generally, the ecological validity of a specific pair of stimuli (Warren, 1977) does not determine whether or not those two stimuli are judged to come from the same object; rather that decision is now under the control of the hierarchy.

The ordering of the levels for identity may be yoked to frequency of occurrence of various behaviors of objects, e.g., repositioning of objects that otherwise maintain their internal structure (isometric) could be the most frequent object transformation and eye movements the most frequent change in the observer while viewing objects. If such were the case, it does not imply that each observer need be a Brunswikian statistician calculating the probabilities of various events in their environments to derive rules of identity. Evolution over the course of the species, rather than development within the individual, would suffice.

However, we suspect the ordering is deeper than frequency and may reflect the recoverability of information from increasingly broad geometries. An analogy is increasingly broad languages and the role they play in language acquisition. According to the subset rule (Berwick, 1985), children assume that an utterance indicates the smallest possible language within which that utterance could be generated and will only adopt a broader language if evidence to the contrary is heard. The logic is that if the broadest language is assumed first, then there would not be any way to proceed from the larger to a smaller language, even if a smaller one were correct; all utterances consistent in the smaller language would also be consistent within the broader language. Consequently, the order of language acquisition proceeds from smaller to larger. The same type of principle may be present for geometry. A given pair of samples is consistent with a entire set of geometries. If the largest is assumed (e.g., topology), then all subsequent transformations of that object, no matter how innocuous, would also be consistent with topology and no transformation would guide one toward a smaller geometry. If a smaller geometry is available, it is advantageous to know it fewer forms are equivalent within smaller geometries and discrimination among more stimuli possible. Otherwise, too many items will get lumped together as "same". More properties are defined in smaller geometries, with a consequent better ability characterize the nature of the object in question. Assuming the smallest geometry is the judicious conclusion and by extension, picking the samples within which identity can be achieved with the smallest geometry, the most conservative option.

Table 2:Transformations. Observer and object transformations for each level geometry. For observer transformations, any affiliated perceptual constancies are noted

\begin{tabular}{lll}
\hline Geometry/ transformation & Observer transformations while looking at object & Transformations to objects themselves \\
$\begin{array}{l}\text { Euclidean/isometric } \\
\text { Similarity }\end{array}$ & $\begin{array}{l}\text { Move eyes left, right, up, down (position constancy) } \\
\text { Walk up to or away from (size constancy) }\end{array}$ & $\begin{array}{l}\text { thrown small, medium-sized solid objects-e.g., a hurled rock } \\
\text { e.g., a (uniformly) squished sponge, a stretched rubber } \\
\text { band, a balloon losing air }\end{array}$ \\
$\begin{array}{ll}\text { Affine } \\
\begin{array}{l}\text { Projective } \\
\text { Topology/topological }\end{array}\end{array}$ & $\begin{array}{l}\text { Turn an angle from }>20 \text { feet away (shape constancy) } \\
\text { Shadows over time of a 4-legged animal while running (?) }\end{array}$ \\
"Non-topology" & Walk around to back of object (?) & $\begin{array}{l}\text { Animacy- e.g., walking, pouncing flexibility-e.g., } \\
\text { bending branches }\end{array}$ \\
& $\begin{array}{l}\text { Accidents of viewing-e.g., } 2 \text { objects line up along line } \\
\text { of sight for one eye, but not other }\end{array}$ & Brobjects cartoons fairy tales \\
\hline
\end{tabular}


Multiple geometries can provide the core foundation of object identity because geometry can be applied before recognizing the type of object involved, can be used in the absence of any instructions, is not specific to one modality (unlike properties such as color) and is applicable to all types of samples. In fact, recent evidence suggests that infants can use geometric properties for object identity before using color or pattern. (Wilcox, 1999). How other properties useful for identity in specific situations interacts with the rules that all situations have in common is not yet clear. Core structure is expected to emerge early in development and persist, exist in other species, exist in all humans of all cultures and provide the building blocks for further abilities (Spelke and Newport, 1998). Perhaps it also can be accessed more quickly, be less prone to errors and be weighted more heavily when it conflicts with other information.

That we may be governed by different geometries at different times and can likely switch between them at lightening speed is exciting. This is especially so given the difficulty humans have in consciously dealing with the formal aspects of just Euclidean geometry. The philosopher Cassirer (1944) recognized 50 years ago that the Klein hierarchy was likely important for perception because of its use of invariance and the progression of the levels towards universality. Yet relatively little use of the hierarchy has been made in psychology. Individual levels have enjoyed great success, as for instance the use of projective geometry for pictures (Cutting, 1988). The hierarchy as a whole has also been used occasionally as a framework for the investigator to use when searching for the right geometry to characterize event perception (Mark and Todd, 1985) or navigation in the rat (Cheng and Gallistel, 1984). Hopefully, the present research will advance the idea that an entire hierarchical structure is available for the same problem, object identity. The problem of non-identical samples dates to Aristotle and non-identical samples show up in all domains. Despite the research that remains, the geometric hierarchy has potential as a general law. Both the general problem and a universal solution run counter to an explosion of research, much of it neuropsychological, that concludes different content areas are modular and independent.

\section{CONCLUSION}

We end on a cautionary note. In theory, the timehonored phenomenon apparent motion is an ideal tool for uncovering the rules of object identity. The ambiguity present in the phenomenon allows one to see how internal rules come into play to resolve the ambiguity. However, apparent motion may be too ambiguous. What observers see is influenced by prior trials, by eye movements, by expectation and by viewing time, with precepts changing over an extended viewing period. Apparent motion may be the Rorshach of experimental psychology. Isolating identity from other influences is painstaking. Object identity and transformation are important. Apparent motion is not.

\section{ACKNOWLEDGEMENT}

Thanks to Karen Wynn, Karen Reinke and Thomas Bever for helpful discussions and Jason Barker for helpful discussions and assistance with figure preparation. Research supported by a grant from the Social and Behavioral Sciences Research Institute (SBSRI) at the University of Arizona.

\section{REFERENCES}

Bedford, F.L., 1999. Keeping perception accurate. Trends Cognit. Sci., 3: 4-11. DOI: 10.1016/S13646613(98)01266-2

Bedford, F.L., 2001. Towards a general law of numerical/object identity. Curr. Psychol. Cognit., 20: 113-175.

Bedford, F.L., 2001. Object Identity Theory and the nature of general laws. Curr. Psychol. Cognit., 20: $277-293$.

Bedford, F.L., 2001. The role of object identity and Klein's geometry in cross-modal and other discrepancies. Curr. Psychol. Cognit., 20: 381-394.

Bedford, F.L., 2001. Generality, mathematical elegance and evolution of numerical/object identity. Brain Behav. Sci., 24: 664-665. DOI: 10.1017/S0140525X01220081

Bedford, F.L., 2003. More on the not-the-liver fallacy: Medical, neuropsychological and perceptual dissociations. Cortex, 39: 170-173. DOI: 10.1017/S0140525X01220081

Bedford, F.L., 2007. Can a space-perception conflict be solved using three sense modalities? Perception, 36: 508-515. DOI: 10.1068/p5632

Berwick, R., 1985. The Acquisition of Syntactic Knowledge. 1st Edn., MIT Press, Cambridge, MA., ISBN: 0262022265, pp: 368.

Bloom, P., 2000. How Children Learn the Meanings of Words. 1st Edn., MIT Press, Cambridge, MA., ISBN: 0262024691, pp: 300.

Burt, P. and G. Sperling, 1981. Time, distance and feature trade-offs in visual apparent motion. Psychol. Rev., 88: 171-195. DOI: 10.1037/0033295X.88.2.171 
Cassirer, E., 1944. The concept of group and the theory of perception. Philosophy Phenomenol. Res., 5: 1-36. DOI: $10.2307 / 2102891$

Chen, L., 1985. Topological structure in apparent motion. Perception, 14: 197-208. DOI: 10.1068/p140197

Cheng, K. and C.R. Gallistel, 1984. Testing the Geometric Power of an Animal's Spatial Representation. In: Animal Cognition, Roitblat, H., T.G. Bever and H. Terrace (Eds.), Lawrence Erlbaum, Hillsdale, New Jersey., ISBN: 0898593344, pp: 409-423.

Dawson, M.R., 1989. Apparent motion and element connectedness. Spatial Vision, 7: 241-251. DOI: 10.1163/156856889X00158

Dennett, D.C., 1996. Darwin's Dangerous Idea: Evolution and the Meanings of Life. 1st Edn., Simon and Schuster, New York ISBN: 0684802902, pp: 586.

Farrell, J.E. and R.N. Shepard, 1981. Shape orientation and apparent rotation motion. J. Exp. Psychol. Hum. Percept. Perform., 7: 477-486. DOI: 10.1037/0096-1523.7.2.477

Green, M., 1986. What determines correspondence strength in apparent motion? Vis. Res., 26: 599-607.

Gibson, J.J., 1966. The Senses Considered as Perceptual Systems. 1st Edn., Houghton-Mifflin, Boston, ISBN: 0313239614, pp: 335.

Goodale, M.A. and A.D. Milner, 1992. Separate visual pathways for perception and action. Trend. Neurosci., 15: 20-25. DOI: 10.1016/01662236(92)90344-8

Goodale, M.A., A.D. Milner, L.S. Jakobson and D.P. Carey, 1991. A neurological dissociation between perceiving objects and grasping them. Nature, 349: 154-156. DOI: $10.1038 / 349154 a 0$

Held, R., 1970. Two modes of Processing Spatially Distributed Information. In: The Neurosciences Second Study Program. Schmitt, F.O. (Ed.). Rockefeller University Press, New York, ISBN: 0874700140, pp: 1068.

Helmholtz, H.E.F. Von, 2005. Treatise on Physiological Optics. Dover Phoenix Edn., Dover Publications, (Original Work Published 1909) New York, ISBN: 0486442640, pp: 496.

Kahneman, D. and A. Treisman and B.J. Gibbs, 1992. The reviewing of object files: Object-specific integration of information. Cognit. Psychol., 24: 175-219. DOI: 10.1016/0010-0285(92)90007-O

Klein, F., 1957. Lectures on Higher Geometry. 3rd Edn., Chelsea. (Original Work Published 1893) New York, pp: 405.
Kolers, P., 1972. Aspects of Motion Perception. 1st Edn., Pergamon Press, Oxford, England, ISBN: 0080168434, pp: 220.

Kolers, P.A. and J.R. Pomerantz, 1971. Figural change in apparent motion. J. Exp. Psychol., 87: 99-108. DOI: $10.1037 / \mathrm{h} 0030156$

Korte, A., 1915. Kinematoscopic investigations. Zeizschrift für Pschologie, 72: 193-296.

Kubovy, M., 1988. Should we resist the seductiveness of the space: Time: Vision: Audition analogy? J. Exp.. Psychol. Hum. Percept. Perform., 14: 318-320. DOI: $10.1037 / 0096-1523.14 .2 .318$

Mack, A., L. Klein, J. Hill and D. Palumbo, 1989. Apparent motion: Evidence of the influence of shape, slant and size on the correspondence process. Percept. Psychophys., 46: 201-206. PMID: 2762108

Mark, L.S. and J.T. Todd, 1985. Describing perceptual information about human growth in terms of geometric invariants. Percept. Psychophys., 37: 249-256. PMID: 4022756

Medin, D.L., R.L. Goldstone and D. Gentner, 1993. Respects for similarity. Psychol. Rev., 100: 254-278. DOI: 10.1037/0033-295X.100.2.254

Modenov and Parkhomenko, 1965. Geometric Transformations. 1st Edn., Academic Press, New York. ISBN: 100125031017, pp: 296.

Mishkin, M., L.G. Ungerleider and K.A. Macko, 1983. Object vision and spatial vision: Two cortical pathways. Trend. Neurosci., 6: 414-417. DOI: 10.1016/0166-2236(83)90190-X

Narter, D.B., 1998. Infants' expectations about the spatial and physical properties of a hidden object. Dissertation Abstracts International: Section B. Sci. Eng., 58(8-B): 4493.

Navon, D., 1976. Irrelevance of figural identity for resolving ambiguities in apparent motion. J. Exp. Psychol. Hum. Percept. Perform., 2: 130-138. DOI: 10.1037/0096-1523.2.1.130

Orlansky, J., 1940. The effect of similarity and difference in form on apparent visual movement. Arch. Psychol., 246: 85.

Rock, I., 1983. The Logic of Perception. 1st Edn., Bradford Books and MIT Press, Cambridge, Mass, ISBN: 0262680459, pp: 384.

Sigman, E. and I. Rock, 1974. Stroboscopic movement based on perceptual intelligence. Perception, 3: 928. DOI: 10.1068/p030009

Shepard, R.N., 1984. Ecological constraints on internal representation: Resonant kinematics of perceiving, imagining, thinking and dreaming. Psychol. Rev., 91: 417-447. DOI: 10.1037/0033-295X.91.4.417 
Shepard, R.N., 2001. Perceptual-cognitive universals as reflections of the world. Brain Behav. Sci., 24: 581-601. DOI: 10.1017/S0140525X01000012

Spelke, E.S. and E.L. Newport, 1998. Nativism, Empiricism and the Development of Knowledge. In: Handbook of Child Psychology: Theories of Development, R.M. Lerner (Ed.)., 5th Edn., Wiley, New York, ISBN: 0471272884, pp: 275-340.

Shepard, R.N. and S.A. Judd, 1976. Perceptual illusion of rotation of three-dimensional objects. Science, 191: 952-954. DOI: 10.1126/science. 1251207

Spelke, E.S., R. Kestenbaum, D. Simons and D. Wein, 1995. Spatio-temporal continuity, smoothness of motion and object identity in infancy. Br. J. Dev. Psychol., 13: 113-142.

Spelke, E.S. and G.A. Van de Walle, 1993. Perceiving and Reasoning about Objects: Insights from Infants. In: Spatial Representation, Eilan, N., W. Brewer and R. McCarthy (Eds.). Basil Blackwell, Oxford, England, ISBN: 0198238878, pp: 426.

Ullman, S., 1979. The Interpretation of Visual Motion. 1st Edn., MIT Press, Cambridge, MA., ISBN: $026221007 X$, p. 244.

Warren, W.H., 1977. Visual information for object identity in apparent movement. Percept. Psychophys., 25: 205-208.
Wertheimer, M., 1961. Experimental Studies on the Seeing of Motion. In: Classics in Psychology: Philosophical Library, Shipley, T. (Ed.). (Original Work 1912) New York, ISBN: 9044665219, pp: 1032-1088.

Wiggins, D., 1980. Sameness and Substance. 1st Edn., Basil Blackwell, Oxford, England, ISBN: 0674785959, pp: 249.

Wilcox, T., 1997. 4.5 and 7.5 Month-old infants' use of shape, color and size when reasoning about object identity. Proceeding of the Biennial Meeting of the Society for Research in Child Development, Washington DC.

Wilcox, T., 1999. Object individuation: Infants' use of shape, size, pattern and color. Cognition, 72: 125-166. DOI: 10.1016/S0010-0277(99)00035-9

$\mathrm{Xu}, \mathrm{F}$. and S. Carey, 1996. Infants' metaphysics: The case of numerical identity. Cognit. Psychol., 30: 111-153. DOI: 10.1006/cogp.1996.0005 